\title{
LONG-TERM STABLE ION SENSITIVE FIELD EFFECT TRANSISTOR SENSORS BASED ON MICROFLUIDICS INDUCED TUNABLE GRAPHENE FILMS
}

\author{
B. Zhang and T. Cui* \\ University of Minnesota, Minneapolis, USA
}

\begin{abstract}
The ion sensitive field effect transistor (ISFET) sensor based on microfluidics induced films is reported in this paper. The graphene film demonstrates controllably tunable thickness different from the conventional graphene composites, and the sensor has a long-term stability in chemical detection. Using capillarity to introduce a graphene suspension solution to a microfluidic system, the graphene film patterns were confined and formed in microchannels. SEM, AFM and Raman spectroscopy were used to analyze the graphene films, demonstrating the tunable thickness of the graphene films in the microchannels. Ambipolar characteristics of the graphene ISFET were also presented. The long-term stability of the ISFET was investigated, and compared with graphene/polymer composites under the same patterning and measurement conditions.
\end{abstract}

\section{INTRODUCTION}

Graphene, a natural two-dimensional structure with only oneatom thick, has attracted more and more attention due to its unique electrical, chemical, and mechanical properties [1-4]. Recently, graphene ion sensitive field effect transistor (ISFET) has been employed to detect various biomolecules, offering advantages including large detection area, relatively low $1 / \mathrm{f}$ noise [5], tunable ambipolar field-effect characteristics, and biocompatibility [6]. Several graphene synthesis approaches were developed, including mechanical exfoliation of graphite [7], epitaxial growth on $\mathrm{SiC}$ or metals [8,9], and chemical vapor deposition growth on metal substrates [10]. However current techniques proposed to generate graphene usually rely on low-yield serial processes typically involving the transfer of optically identified exfoliated graphene or graphene grown on metal substrates, which requires highly trained professionals and high cost. After the synthesis and preparation, graphene films have been extensively integrated with other materials such as polymers to obtain various structures with superior strength, flexible transparent and conductive films [11] to extend their good properties for specific applications. However, most of these graphene composites are not capable of turning or controlling their structures owing to their fixed structural synthesis processes. In addition, the integrated materials in the graphene composite structures damage the electrical stability of pure graphene, introducing an electrical drift in sensing applications. To overcome these hurdles of previous graphene synthesis and applications, we present a simple and low-cost approach to form graphene films, using microfluidics to introduce, confine and pattern the graphene films for ISFETs. This developed graphene films were tunable in thickness due to the controllable height of microchannels. The graphene was demonstrated with long-term stability in ISFET sensors, compared with the graphene/polymer composite with the same patterns and measurement conditions. Therefore, this tunable microfluidics induced graphene films are expected to offer more controllable and flexible applications to chemical detection and microfluidics.

\section{DESIGN AND FABRICATION}

The fabrication process of microfluidics induced graphene films is illustrated in Fig. 1. Microfabrication was utilized to build the microchannels, and microfluidics was used to deposit the graphene films. First, a layer of photoresist (Shipley S1813) was spin coated on silicon wafers with silicon dioxide $300 \mathrm{~nm}$ thick, followed by a patterning of photoresist with photolithography. Subsequently, the patterned substrate was immersed into HF buffer solution (10:1) to obtain the microchannel array in the silicon dioxide layer. To control the etching time, different channel heights from $50 \mathrm{~nm}$ to $250 \mathrm{~nm}$ were generated. After striping the photoresist, research grade graphene (PureSheets ${ }^{\mathrm{TM}}$, Nanointegris Inc, $0.25 \mathrm{mg} / \mathrm{ml}$ ) suspension solution was introduced into the reservoir. Due to the hydrophilic property of silicon dioxide, the capillarity induced the graphene suspension into the microchannel array. Next, the whole system was placed in a vacuum oven (Model 280A) for $30 \mathrm{~min}$ to degas and dry up the solution at room temperature under vacuum condition. The extra graphene films in the reservoir were gently scraped off by a blade. Chromium/gold layers $10 / 100 \mathrm{~nm}$ thick were deposited at the two ends of the microchannels with an AJA sputter system (Model ATC 2000), and sensor electrodes were patterned by photolithograph with the same photoresist.
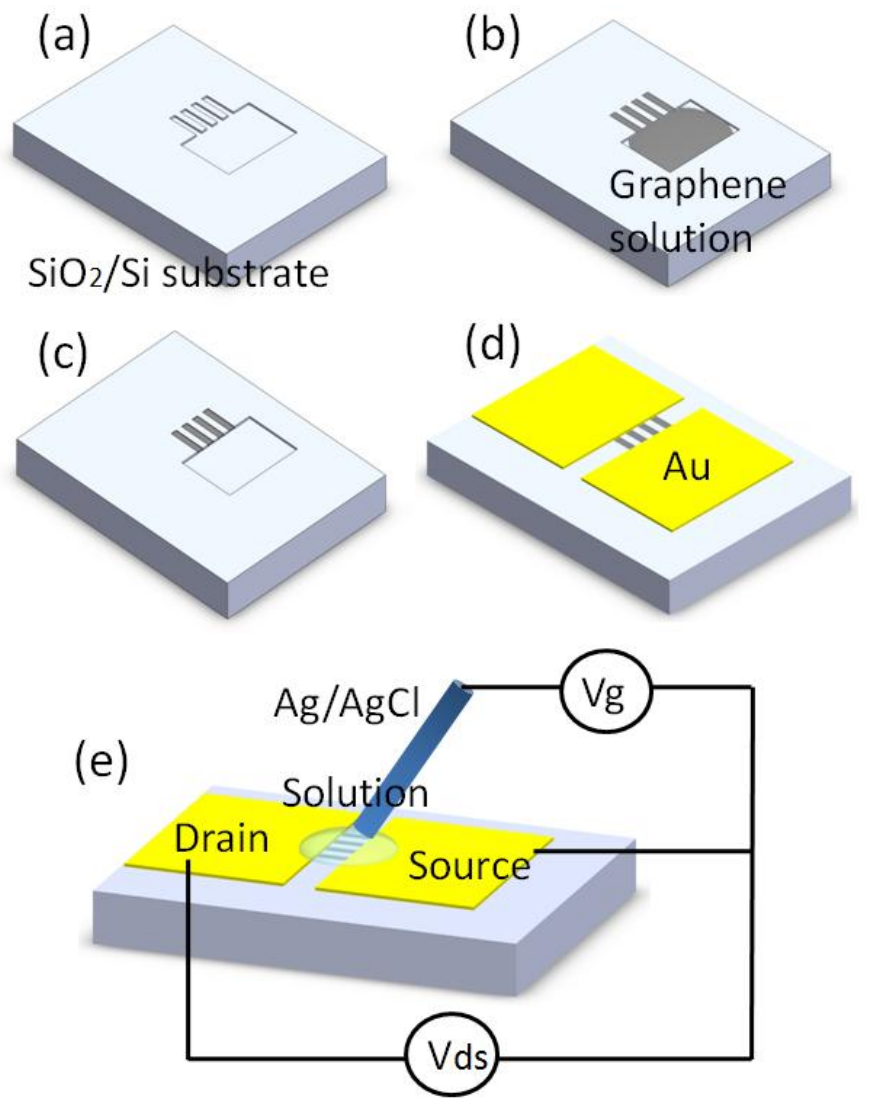

Figure 1: (a) Microchannels were patterned on a substrate; (b) Introduce graphene solution; (c) Remove the extra graphene films in reservoir; (d) Deposit and pattern electrodes; (e) Sketch of the microfluidics induced graphene ISFET. Target solutions were introduced onto the graphene films, and an $\mathrm{Ag} / \mathrm{AgCl}$ reference electrode was immersed to apply a desired gate. 
Fig. 1(e) demonstrates the structure of the microfluidics induced graphene ISFET. Target solutions were introduced onto the graphene films, and an $\mathrm{Ag} / \mathrm{AgCl}$ reference electrode was immersed to apply a desired gate voltage. Electrical measurements were carried out using a semiconductor device analyzer (HP 4145B). The microfluidics induced graphene films were also inspected by scanning electron microscope (SEM) and atomic force microscope (AFM). As shown in Fig. 2(a), the microchannel array was fabricated in the silicon dioxide layer on a silicon substrate. Two electrodes were deposited in the microchannel to form the ISFET structure, as shown in Fig. 2(b). Fig. 2(c) and 2(d) present the porous surface profile of microfluidics induced graphene, and the graphene nanoplatelets were deposited randomly across the channel of two electrodes. Besides, the samples of graphene films deposited in microchannels were studied by Raman mapping $(100 \times 100$ pixels, Witec Alpha300R), and G peak and 2D band mapping results were presented in Fig 3(a) and 3(b), respectively. The intensity difference of the $G$ peak and $2 \mathrm{D}$ band was clearly shown between internal and external channels, which proved that the graphene film patterns were successfully deposited and confined by microchannels.
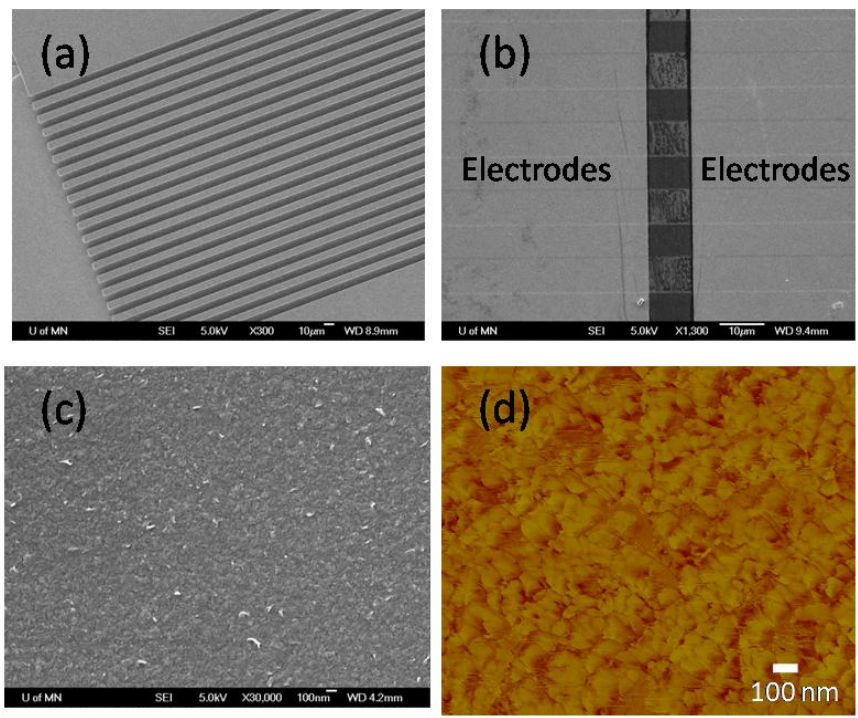

Figure 2: SEM images of (a) microchannel array, (b) ISFET structure with two electrons deposited at both ends of the microchannels, and (c) porous surface profile of microfluidics induced graphene films. (d) AFM image of the graphene nanoplatelets deposited randomly across the channel of two electrodes.

\section{RESULTS AND DISCUSSION}

AFM was used to characterize the thickness of graphene films in different microchannels with various heights. To control the etching time of silicon dioxide in HF buffer solution, the heights of microchannels were obtained from $65 \mathrm{~nm}$ to $221 \mathrm{~nm}$. After degasing and drying up in a vacuum oven, the thickness of the deposited graphene films was measured in the microchannels with different heights. As shown in Fig. 4, the thickness of the graphene films were raising with the increase of the microchannel heights. This can be explained that different amounts of graphene suspension were introduced by capillarity with various channel heights. As a result, the thickness of the graphene films is capable of tuning by adjusting the height of microchannel.
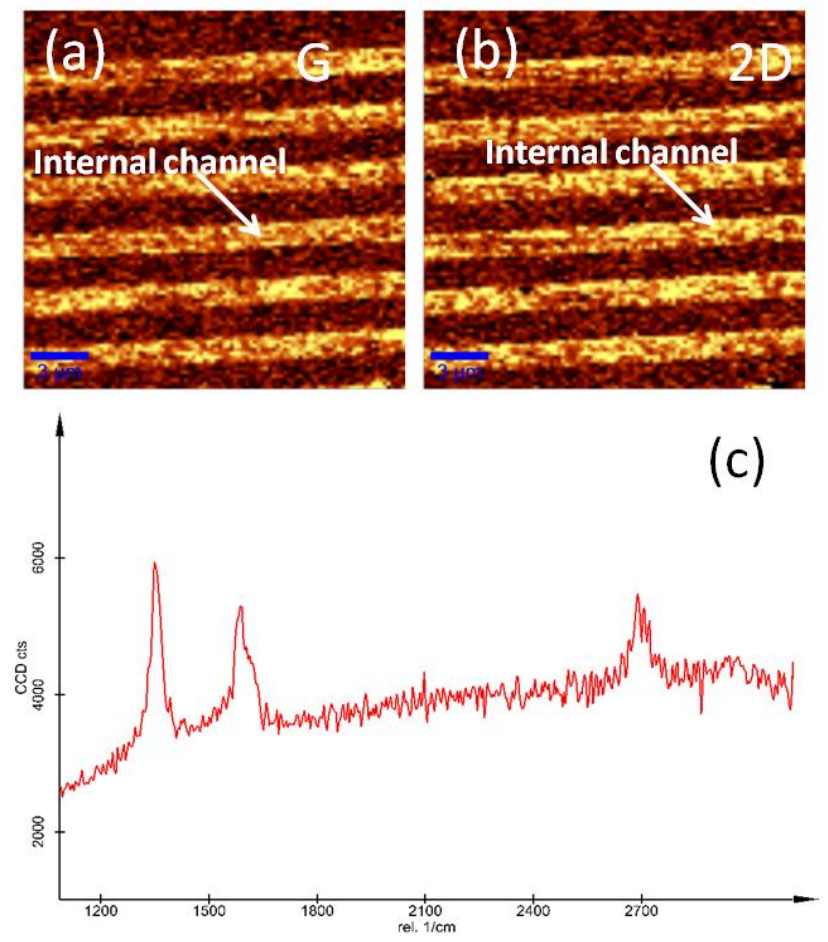

Figure 3: (a) $G$ peak and (b) 2D band Raman spectroscopy mapping results. (c) Typical Raman spectra of microfluidics induced graphene films. The intensity difference of the $G$ peak and $2 D$ band was clearly shown between internal and external channels, which proved that the graphene film patterns were successfully deposited and confined by microchannels.

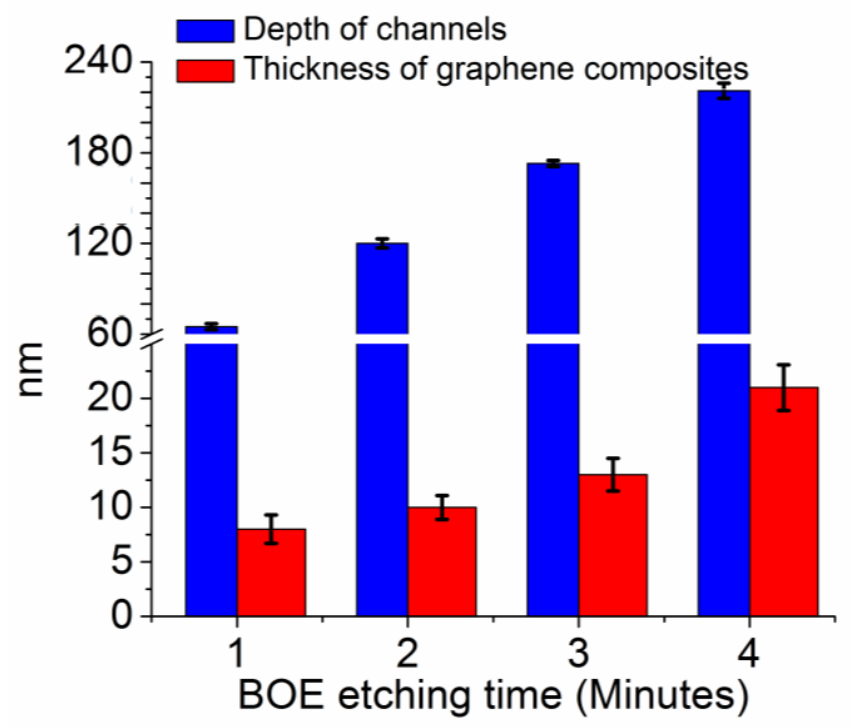

Figure 4: AFM was used to characterize the thickness of graphene films in different microchannels with various heights. The thickness of the graphene films increases with the increase of the microchannel heights.

Drain-to-source current versus solution gate voltage were recorded to investigate the electrical properties of microfluidic induced graphene ISFET. Fig. 5(a) presents ambipolar characteristics of the graphene ISFET measured in PBS buffer solution (Dulbecco's phosphate buffered saline, Invitrogen Inc.) at 
room temperature, showing the transition from $\mathrm{p}$-type region to $\mathrm{n}$ type region at the Dirac point. The electrolyte gate response of the ISFET's drain-to-source current $\left(\mathrm{I}_{\mathrm{d}}\right)$ in different $\mathrm{pH}$ solutions were also recorded. As shown in Fig. 5(b), solutions from $\mathrm{pH} 6$ to $\mathrm{pH} 9$ were delivered to ISFET sequentially. While the ISFET was biased at $\mathrm{V}_{\mathrm{ds}}$ at $2 \mathrm{~V}$, and the gate voltage was applied from -4.5 to $4.5 \mathrm{~V}$. The Dirac point of graphene ISFET was obviously observed to shift positively from $\mathrm{pH} 5$ to $\mathrm{pH} 9$, suggesting that $\mathrm{H}^{+}$ ions act as n-type dopants to the graphene. Adsorption of a positively charged $\mathrm{H}^{+}$induces additional negative charge in the graphene, thus n-type doping the graphene and shifting the Dirac point toward more negative gate voltages. The graphene films behave as a p-type material when negative gate potential is applied, and the $I_{d}$ of the ISFET increases with the increase of $\mathrm{pH}$ values. On the contrary, when the gate potential is switched positive, transition from $\mathrm{p}$-type region to n-type region occurs, and the $\mathrm{I}_{\mathrm{d}}$ of the ISFET decreases with increased $\mathrm{pH}$.
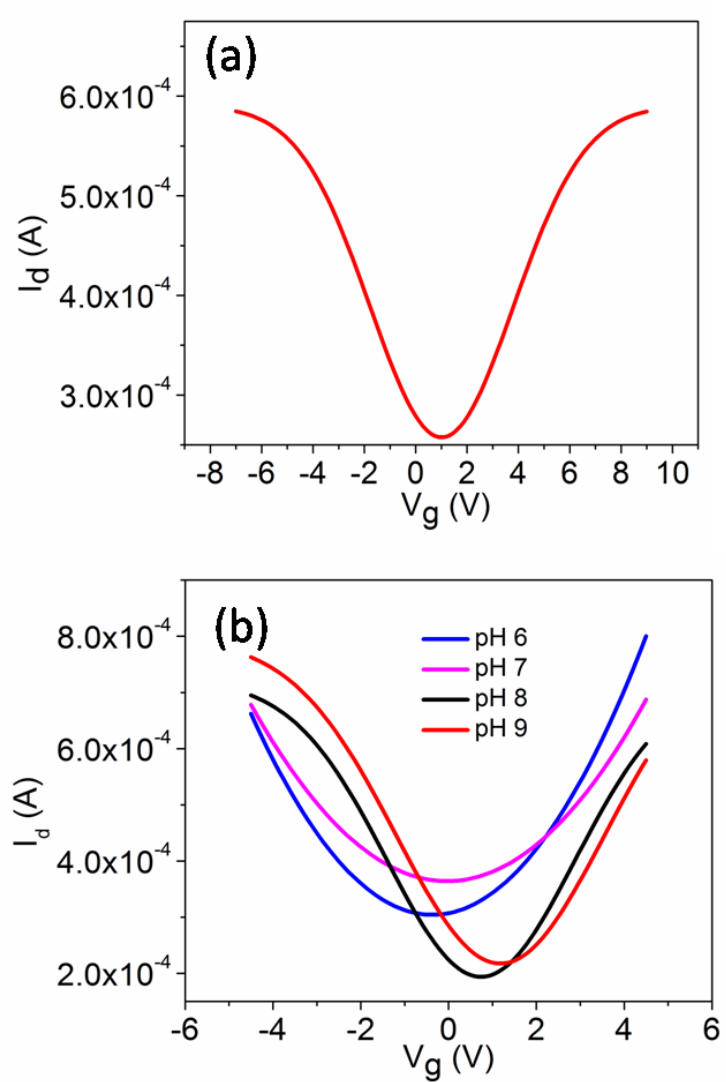

Figure 5: (a) Ambipolar characteristics of the graphene ISFET measured in a PBS buffer solution at room temperature; (b) Solutions from $\mathrm{pH} 6$ to $\mathrm{pH} 9$ were delivered to ISFET sequentially. Adsorption of a positively charged $H^{+}$induces additional negative charges in the graphene, thus n-type the graphene and shifting the Dirac point toward more negative gate voltages.

The ISFET was also verified for functionality and detect limitation of glucose sensing. After the graphene ISFET was fabricated, glucose oxidase (GOx) was immobilized on the sensing region. The GOx on the surface reacted with the glucose and created hydrogen ions as such:

$$
\begin{aligned}
& \beta-D-\text { glucose }+\mathrm{O}_{2} \stackrel{\text { GOx }}{\longrightarrow} D-\text { glucono- }-\delta \text {-lactone }+\mathrm{H}_{2} \mathrm{O}_{2} \\
& D-\text { glucono- } \delta \text {-lactone }+\mathrm{H}_{2} \mathrm{O} \rightarrow D-\text { gluconate } \\
& +
\end{aligned}
$$

As demonstrated previously, the graphene ISFET was capable of responding to the $\mathrm{H}^{+}$. Different concentrations of glucose can cause different local pH. As shown in Fig. 6(a), different concentrations of glucose solutions were delivered to the graphene ISFET. The $\mathrm{V}_{\mathrm{ds}}$ was biased at $2 \mathrm{~V}$, and the gate voltage was kept at $4 \mathrm{~V}$. The current from source to drain was measured at different concentrations of glucose, demonstrating the detection limits of graphene ISFET down to $400 \mathrm{pM}$.
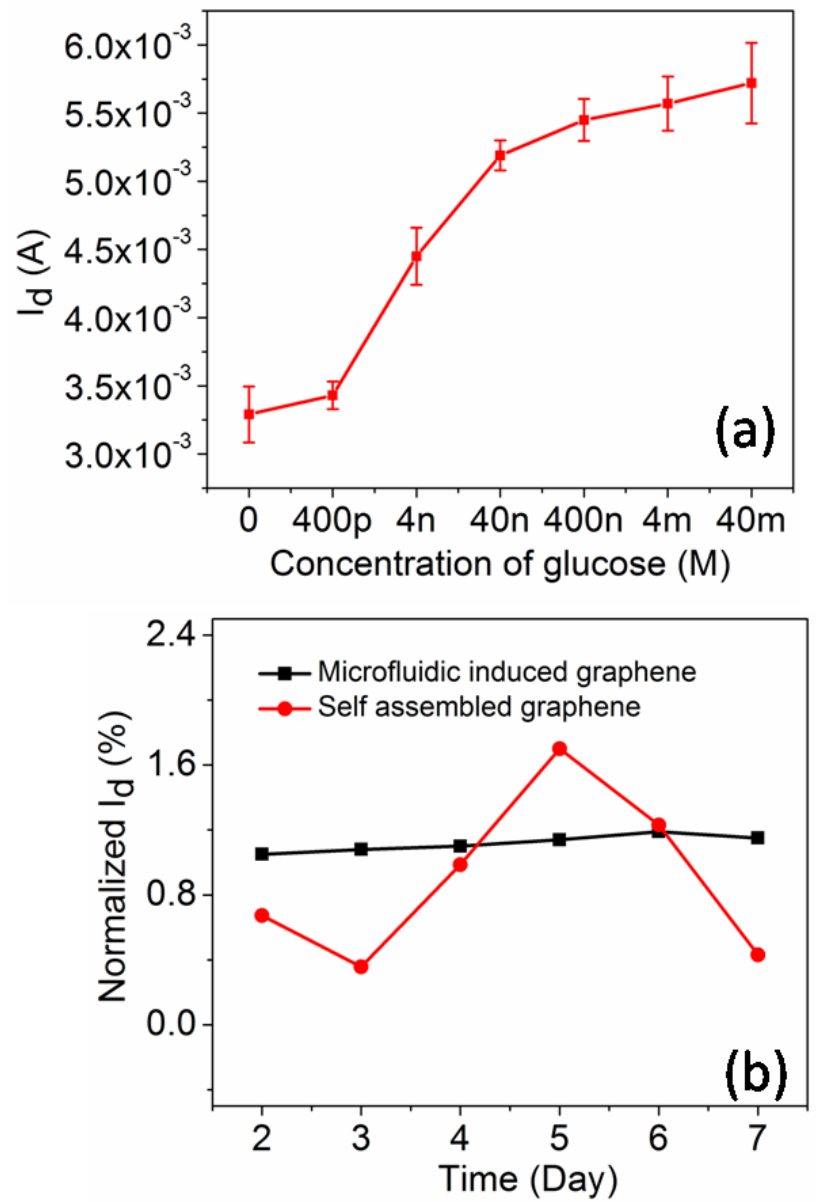

Figure 6: (a) Glucose detection was characterized on an ISFET modified by GOx, presenting a detection limit down to $400 \mathrm{pM}$. (b) Microfluidics induced graphene ISFET was much more stable than the self assembled graphene ISFET. The absence of polymer or other materials in the microfluidics induced graphene may be less sensitive to the disturbance of the environments, compared with the composite graphene structures.

The long-term stability of the graphene ISFET was investigated, and compared with layer-by-layer self assembled graphene ISFET. The fabrication processes of self assembled graphene were reported in our previous work [12]. Two types of graphene ISFET under same patterning and testing conditions were used to measure the response of $I_{d}$ for $\mathrm{pH} 7$ solutions in 7 days continuously. During the idle time, the ISFETs were kept in DI water to prevent from contamination and disturbance. Both of the ISFETs were biased at $\mathrm{V}_{\mathrm{ds}}$ of $1 \mathrm{~V}$, and the gate voltage was fixed at $2 \mathrm{~V}$. To obtain more clear results, a normalized $I_{d}$ was introduced. $I_{d}$ in the first day result was used as an initial current $I_{0}$. Normalized $I_{d}$ was represented as the ratio of the following day result to the initial current $\left(\mathrm{I}_{\mathrm{d}} / \mathrm{I}_{0}\right)$. As shown in Fig. 6, the microfluidics induced graphene ISFET was much more stable than 
the self assembled graphene ISFET. The absence of polymer or other materials in the microfluidics induced graphene may be less sensitive to the disturbance of the environments, compared with the graphene composite structures.

\section{CONCLUSIONS}

In summary, the microfluidics induced graphene films offer a number of advantages over the current graphene composites. Due to controllable microchannel heights, the graphene thickness is tunable, superior to that of general macroscopic graphene composites with fixed structures. Using a microfluidic system as a fabrication platform, the graphene ISFET should be low cost, and easy to integrate with microfluidic system for many biomedical applications. In addition, owing to the absence of other materials in its structure, this graphene ISFET exhibits long-term stability for various chemical detections. This microfluidics induced graphene films can be a general strategy to fabricate a broad class of macroscopic graphene structures with tunable properties and longterm stability.

\section{ACKNOWLEDGEMENTS}

The authors acknowledge the assistance of fabrication and characterization from the Nanofabrication Center and the Characterization Facility at the University of Minnesota.

\section{REFERENCES}

[1] K. S. Novoselov, A. K. Geim, S. V. Morozov, D. Jiang, Y. Zhang, S. V. Dubonos, I. V. Grigorieva, A. A. Firsov, "Electric Field Effect in Atomically Thin Carbon Films", Science, 306, 666 (2004).

[2] K. S. Novoselov, A. K. Geim, S. V. Morozov, D. Jiang, M. I. Katsnelson, I. V. Grigorieva, S. V. Dubonos, A. A. Firsov, "Twodimensional Gas of Massless Dirac Fermions in Graphene", Nature, 438, 197 ( 2005).
[3] A. A. Balandin, S. Ghosh, W. Bao, I. Calizo, D. Teweldebrhan, F. Miao, C. Lau, "Superior Thermal Conductivity of Single-Layer Graphene", Nano Letters. 8, 902 (2008).

[4] C. Lee, X. D. Wei, J. W. Kysar, J. Hone, "Measurement of the Elastic Properties and Intrinsic Strength of Monolayer Graphene", Science, 321, 385 (2008).

[5] K. Ratinac, W. Yang, S. Ringer, F. Braet, "Toward Ubiquitous Environmental Gas Sensors-Capitalizing on the Promise of Graphene", Environmental Science Technology 44, 1167 (2010).

[6] S. Agarwal, X. Z. Zhou, F. Ye, Q. Y. He, G. C. K. Chen, J. Soo, F. Boey, H. Zhang, P. Chen, "Interfacing Live Cells with Nanocarbon Substrates", Langmuir, 26, 2244 (2010).

[7] P. Li, Z. You, G. Haugstad, T. Cui, "Graphene Fixed-End Beam Arrays based on Mechanical Exfoliation", Applied Physics Letter, 98, 253105 (2011).

[8] K. S. Kim, Y. Zhao, H. Jang, S. Y. Lee, J. M. Kim, K. S. Kim, J.-H. Ahn, P. Kim, J.-Y. Choi, B. H. Hong, "Large-Scale Pattern Growth of Graphene Films for Stretchable Transparent Electrodes", Nature 457, 706 (2009).

[9] C. Berger, Z. M. Song, X. B. Li, S. Wu, X. N. Brown, C. Naud, D. Mayou, T. B. Li, J. Hass, A. N. Marchenkov, "Electronic Confinement and Coherence in Patterned Epitaxial Graphene", Science, 312, 1191 (2006).

[10] M. P. Levendorf, C. S. Ruiz-Vargas, S. Garg, J. Park, "Transfer-Free Batch Fabrication of Single Layer Graphene Transistors", Nano Letters. 9, 4479 (2009).

[11] Z. Chen,W. Ren, L.Gao, B. Liu, S. Pei, H.Cheng, "ThreeDimensional Flexible and Conductive Interconnected Graphene Networks Grown by Chemical Vapour Deposition", Nature Materials, 10, 424, (2011).

[12] B. Zhang, T. Cui, "An Ultra-Sensitive and Low-Cost Graphene Sensor Based on Layer-by-Layer Nano Self-Assembly", Applied Physics Letter, 98, 073116 (2011).

\section{CONTACT}

*T. Cui, tel: +1-612-626-1636; tcui@me.umn.edu 